\title{
Self-recoverable semi-crystalline hydrogels with thermomechanics and shape memory performance
}

\author{
Kangjun Zhang ${ }^{1}$, Ziguang Zhao ${ }^{1}$, Jin Huang ${ }^{1}$, Tianyi Zhao ${ }^{1}$, Ruochen Fang ${ }^{1,3^{*}}$ and Mingjie Liu ${ }^{1,2,3^{*}}$
}

\begin{abstract}
Stimuli-responsive hydrogels have become one of the most popular artificial soft materials due to their excellent adaption to complex environments. Thermoresponsive hydrogels triggered by temperature change can be efficiently utilized in many applications. However, these thermoresponsive hydrogels mostly cannot recover their mechanical states under large strain during the process. Herein, we utilize the heterogeneous comb-type polymer network with semicrystalline hydrophobic side chains to design self-recovery semi-crystalline hydrogels. Based on hydrophilic/hydrophobic cooperative complementary interaction and heterogeneous polymer network, hydrogels can be endowed with excellent thermosensitive properties and mechanical performance. The hydrogels exhibit high compressive strength (7.57 MPa) and compressive modulus (1.76 MPa) due to the semi-crystalline domains formed by association of the hydrophobic poly ( $\varepsilon$-caprolactone) PCL. The melting-crystalline transition of PCL and elastic polymer network provide the hydrogels excellent thermomechanical performance and self-recovery property. Furthermore, the hydrogels exhibit shape memory behavior, which can be realized by simple process and smart surface patterning. With these excellent properties, our hydrogels can be applied in sensors, flexible devices and scaffolds for tissue engineering.
\end{abstract}

Keywords: semi-crystalline hydrogels, thermomechanics, selfrecovery, shape memory property

\section{INTRODUCTION}

Hydrogels are ideal soft materials which can substitute living tissues due to their biocompatibility and "wet and soft" natures [1-3]. Stimuli-responsive hydrogels can switch their physicochemical properties in response to environmental stimuli (i.e., $\mathrm{pH}$, heat, ionic strength, magnetic/electric fields) [4-10], and thus have attracted considerable attention for many applications, such as drug delivery systems, soft robots and sensors [11-13]. For example, poly( $N$-isopropylacrylamide) (PNIPAM) is known as a thermosensitive polymer with a lower critical solution temperature (LCST) at $32^{\circ} \mathrm{C}$. PNIPAM hydrogels can be tuned for their transparency, swelling ratio and mechanical performance by thermal stimuli $[14,15]$. These conventional PNIPAM covalent hydrogels are mostly mechanically weak, and suffer unstable shape during their responsive process, which limits their further application in the complex environments. A strategy for improving mechanical properties is utilizing the reversible non-covalent interactions such as hydrogen bonding [16], electrostatic interaction [17], hydrophobic associations [18] and crystalline segments [19] serving as dynamic physical polymer netpoints. For example, Liu et al. [20] designed supramolecular poly $(N$-acryloyl glycinamide) hydrogels based on dual hydrogen bonding interactions, which had thermoplastic and self-healable properties. Zhang et al. [21] utilized dipole-dipole and $\mathrm{H}$-bonding interaction to design a thermosensitive shape memory hydrogel by copolymerization of acrylonitrile (AN), acrylamide (AAm), and 2-acrylamido-2-methyl-1propanesulfonic acid (AMPS). In addition to the reversible non-covalent interactions, the hydrophilic/ hydrophobic cooperative complementary interaction can also efficiently improve the mechanical properties and provide excellent thermo-responsiveness [22-24]. The micelles polymerization technology and block copolymer systems are usually used to introduce the hydrophobic polymer in the hydrophilic polymer network. Okay and co-workers [25-27] have prepared thermomechanical hydrogels by micellar copolymerization technique due to

\footnotetext{
${ }^{1}$ Key Laboratory of Bio-Inspired Smart Interfacial Science and Technology of Ministry of Education, School of Chemistry, Beihang University, Beijing 100191, China

${ }^{2}$ International Research Institute for Multidisciplinary Science, Beihang University, Beijing 100191, China

${ }^{3}$ Beijing Advanced Innovation Center for Biomedical Engineering, Beihang University, Beijing 100191, China

* Corresponding authors (emails: liumj@buaa.edu.cn (Liu M); fangruochen@buaa.edu.cn (Fang R))
} 
the melt-crystallization properties of micelles. Ding et al. [28-32] have developed thermoresponsive physical hydrogels with amphiphilic block copolymers composed of hydrophilic PEG and hydrophobic biodegradable polyesters. However, these strategies for fabricating thermoresponsive hydrogels mostly depend on complex chemical synthesis. Meanwhile, these thermoresponsive hydrogels mostly cannot recover their mechanical states under large strain during the process. Therefore, new methods need to be developed to prepare thermoresponsive hydrogels with excellent self-recoverable property.

Herein, based on the hydrophilic/hydrophobic cooperative complementary interaction, we utilize the heterogeneous comb-type polymer network in which the poly(e-caprolactone) (PCL) serve as the semi-crystalline side chains to design self-recoverable semi-crystalline hydrogels (SCHs) with excellent thermomechanical performance and shape memory property. In the SCHs, the hydrophilic $N, N$-dimethylacrylamide (DMA) and 2-methoxyethyl acrylate (MEA) form the hydrophilic backbones, and semi-crystalline PCL side chains associate to form rigid domains. The obtained stable SCHs exhibit high compressive strength $(7.57 \mathrm{MPa})$ and compressive modulus $(1.76 \mathrm{MPa})$. The efficient energy dissipation of heterogeneous polymer network gives high toughness $\left(208 \mathrm{~kJ} \mathrm{~m}^{-3}\right)$ to the SCHs. Due to excellent restoring ability of the heterogeneous polymer network at temperature above the $T_{\mathrm{m}}$ of the PCL, the self-recovery properties of $\mathrm{SCH}$ s can be realized by thermo stimuli. Meanwhile, both the SCHs and dry polymer network exhibit shape memory effects. Combining above excellent properties, our $\mathrm{SCH}$ can be applied in flexible devices and biological materials.

\section{EXPERIMENTAL SECTION}

\begin{abstract}
Materials
$\varepsilon$-Caprolactone (CL), triethylamine, 2,2'-diethoxy-acetophenone (DEOP) and methacryloyl chloride were purchased from Acros Organics. MEA, benzyl alcohol, tin(II) 2-ethylhexanoate $\left(\mathrm{Sn}(\mathrm{Oct})_{2}\right)$ were supplied by Alfa Aesar. Dichloromethane and methanol were obtained from Sinopharm, China. DMA was obtained from Sigma-Aldrich. All the chemicals were used without further purification.
\end{abstract}

\section{Synthesis of poly(e-caprolactone) (PCL-OH)}

$\mathrm{PCL}-\mathrm{OH}$ was synthesized by ring-opening polymerization of CL using benzyl alcohol as an initiator and $\mathrm{Sn}(\mathrm{Oct})_{2}$ as the catalyst. Benzyl alcohol (2.106 g, $0.0195 \mathrm{~mol})$, CL $(40 \mathrm{~g}, 0.351 \mathrm{~mol})$ and $\mathrm{Sn}(\mathrm{Oct})_{2}(0.12 \mathrm{~g}, 0.3 \mathrm{wt} \%$ of CL) were added to a Schlenk flask, and then were kept at $110^{\circ} \mathrm{C}$ for $12 \mathrm{~h}$ after three freeze-pump-thaw cycles. After cooling to room temperature, the resulting polymer was dissolved in dichloromethane and precipitated in excess cold methanol three times. The white precipitate was obtained and dried under vacuum at room temperature for $48 \mathrm{~h}$. The yield of PCL-OH is $90 \%$.

Synthesis of poly( $\varepsilon$-caprolactone) methacrylate (PCL-MA) PCL-MA was obtained by the reaction of PCL-OH with excess triethylamine and methacryloyl chloride. PCL-OH $\left(M_{\mathrm{n}}=2,000,8 \mathrm{~g}, 3.9 \mathrm{mmol}\right)$ and triethylamine $(3.151 \mathrm{~g}$, $31.2 \mathrm{mmol}$ ) were dissolved in $100 \mathrm{~mL}$ dichloromethane in a three-necked flask under ice bath. After half an hour, methacryloyl chloride $(4.075 \mathrm{~g}, 39 \mathrm{mmol}$ ) was added to the reaction systems and the reaction was carried on under room temperature for $24 \mathrm{~h}$. After the reaction, the solution was concentrated under reduced pressure to obtain sticky solution. Next, the sticky solution was dissolved in dichloromethane and precipitated in excess cold methanol three times. The precipitate was dried under vacuum at room temperature for $48 \mathrm{~h}$, and the flaxen PCL-MA was obtained with a yield of $92 \%$.

\section{Synthesis of the stimuli-responsive SCHs and corresponding dry polymer network}

Firstly, 1 g PCL-MA (4000), 0.5 g DMA, 0.5 g MEA and $5 \mathrm{mg}$ DEOP were heated and mixed, and then, poured into a mold. The dry polymer network was synthesized by ultraviolet photo-initiated polymerization at room temperature for $40 \mathrm{~min}$. Next, the $\mathrm{SCH}_{2.5-4000}$ was prepared by swelling the dry polymer network for $60 \mathrm{~h}$, in which 2.5 is the mole ratio of the oleophilic PCL relative to the total hydrophilic monomers and 4,000 is the molecular weight of the PCL. In all SCHs, the weight ratio of DMA and MEA in the hydrophilic components was kept the same (5:5). When considering different molecular weight of PCL $(2,000,4,000,6,000$, see Table S1), the hydrophilic monomers and the mole ratios between hydrophilic components and hydrophobic components were kept the same (100:2.5). The components of different SCHs are listed in Table S2 in detail.

\section{Synthesis of the hydrogel with hydrophilic polymer backbone}

$0.5 \mathrm{~g}$ DMA, $0.5 \mathrm{~g}$ MEA and $5 \mathrm{mg}$ DEOP were mixed and polymerized by ultraviolet light at room temperature for $40 \mathrm{~min}$. Next, the hydrogel was prepared by swelling the 
a<smiles>C=CC(=O)N(C)C</smiles><smiles>C=CC(=O)OCCOC</smiles>

2-Methoxyethyl

$N, N$-dimethyl acrylamide

acrylate

(DMA)

(MEA)<smiles>C=C(C)C(=O)OCCCCCC(=O)OCc1ccccc1</smiles>

Poly (s-caprolactone)

methacrylate (PCL-MA)

b

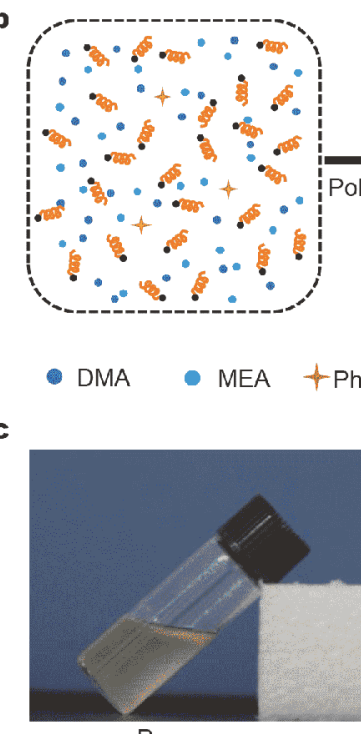

Precursor
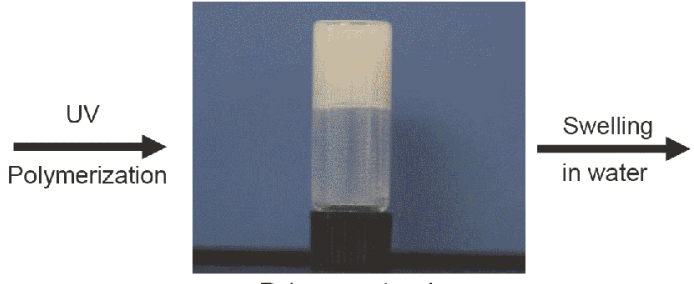

Polymer networks

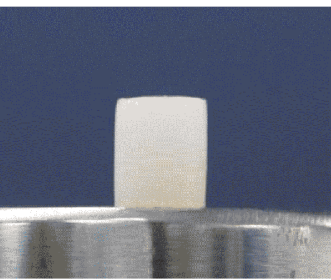

Hydrogels
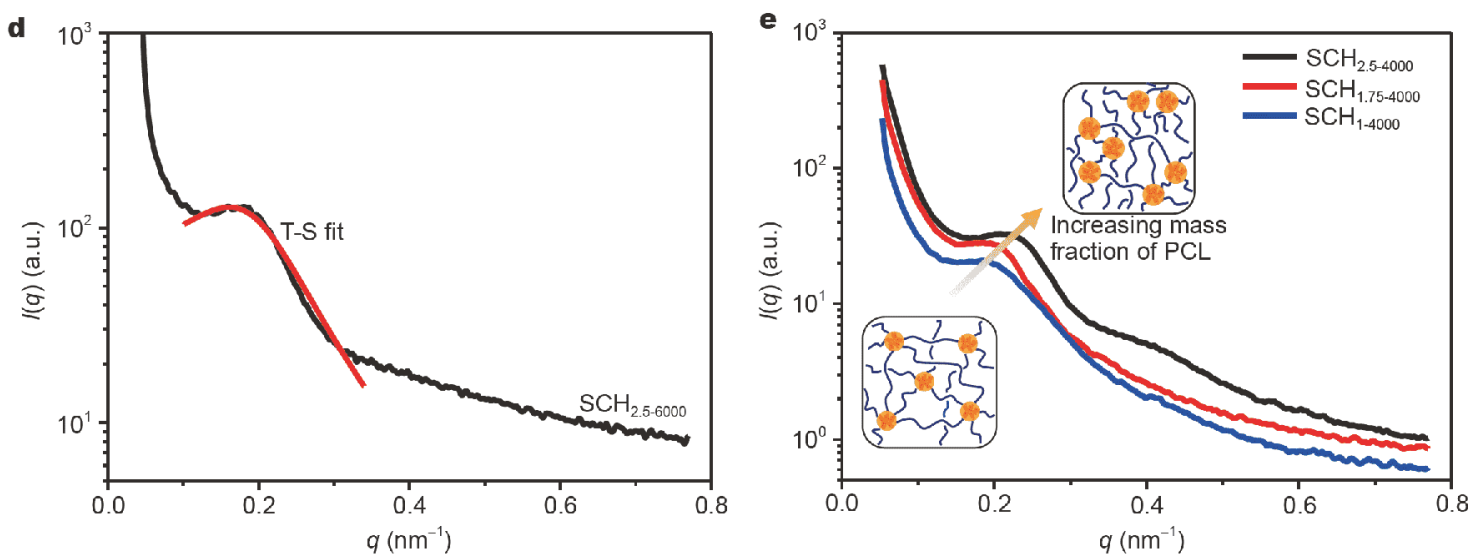

Figure 1 Preparation of SCHs. (a) Molecular structure of DMA, MEA and PCL-MA. (b) Schematic illustration of SCHs prepared by UV light polymerization and subsequently swelling. (c) The photos of precursor solution, polymer networks and SCHs. (d) The fitting of SAXS data of $\mathrm{SCH}_{2.5-6000}$ by T-S model. (e) SAXS profiles of SCHs with different PCL contents.

network for $60 \mathrm{~h}$.

\section{RESULTS and DISCUSSION}

\section{Compositions and structures of hydrogels}

In our work, we fabricated heterogeneous comb-type polymer networks through an in-situ UV polymerization strategy. Firstly, PCL-M) as the macromonomers was synthesized by ring-opening polymerization and reaction with methacryloyl chloride and triethylamine (Fig. 1a and Fig. S1). The PCL is a representative semi-crystalline, biodegradable and biocompatible polymer, which has 

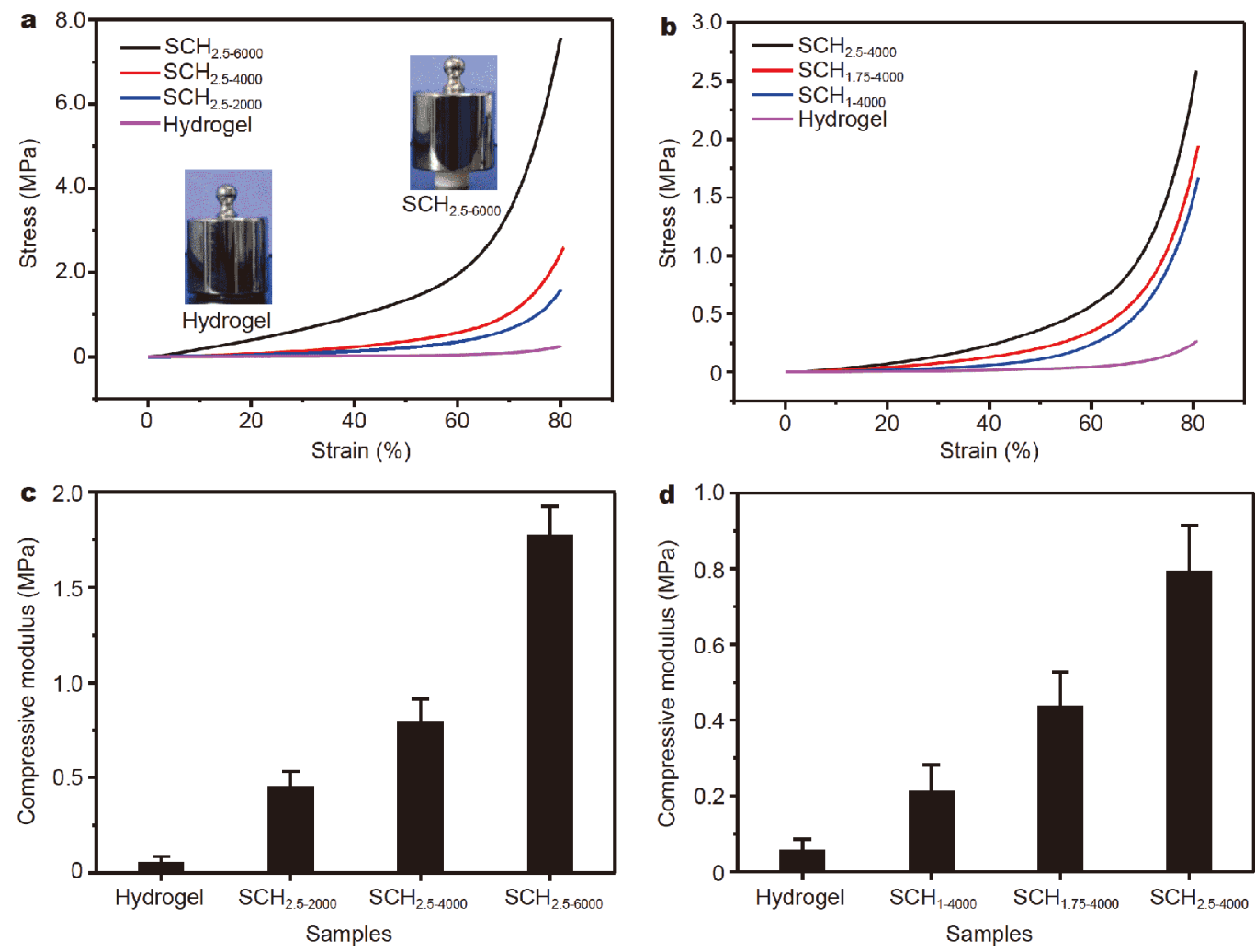

Figure 2 Mechanical properties of SCHs. (a) Compressive strain-stress curves of hydrogel and SCHs with different PCL molecular weights. (b) Compressive strain-stress curves of hydrogel and SCHs with different PCL contents. (c, d) Compressive modulus of hydrogel, SCHs with different molecular weights and SCHs with different PCL contents, respectively.

been widely applied in tissue engineering and drug delivery [33-36]. Next, as shown in Fig. 1b, the hydrogel precursors which consisted of DMA, MEA, PCL-MA and photoinitiator DEOP formed the heterogeneous combtype polymer networks. These small molecular monomers can form the hydrophilic backbones, and oleophilic PCL macromonomers serve as functional side chains with stimuli-responsive semi-crystallinity. During the infiltration process in aqueous phase, our comb-type polymer network can be further swollen to form elastic hydrogel with the stimuli-responsive semi-crystallinity (Fig. 1c). In our system, the hydrophilic network backbones act as elastic framework, and PCL side chains can aggregate to form rigid micro-domains due to the semi-crystallization effect and hydrophobic interactions. Based on such heterogeneous network, our stimuli-responsive SCHs exhibit high mechanical strength, thermomechanical property, self-recovery performance and shape memory behaviors. Meanwhile, through accurate and independent design of the degree of polymerization (DP) of the PCL side chains in heterogeneous network, we can realize the precise control for the specific properties of our hydrogel mate- rials.

During the swelling process of the comb-type polymer network, our materials exhibited highly self-constrain behavior due to the heterogeneous amphiphilic feature between network backbones and side chains. In our hydrogel network, the network backbones can swell due to their hydrophilic natures. These PCL side chains form rigid domains because of their semi-crystallization and hydrophobic association, which further limits the swelling degree of heterogeneous network. In our case, we investigated the swelling behaviors of our SCHs with different contents of oleophilic polymer side chains. The hydrogels are denoted as $\mathrm{SCH}_{a-b}$, where $a$ is the mole ratio of the oleophilic PCL relative to the total hydrophilic monomers and $b$ is the molecular weight of the PCL. The detail components of hydrogels are listed in Table S2. Fig. S3 shows the swelling performance of the SCHs, which demonstrates that the swelling ratios of SCHs are strongly relative to the PCL contents. The water contents of swelling equilibrium decreased with increasing PCL contents from $230 \%$ of $\mathrm{SCH}_{1-4000}$ to $146 \%$ of $\mathrm{SCH}_{2.5-4000}$. Fig. S4 shows the photos of the volume of the SCHs 

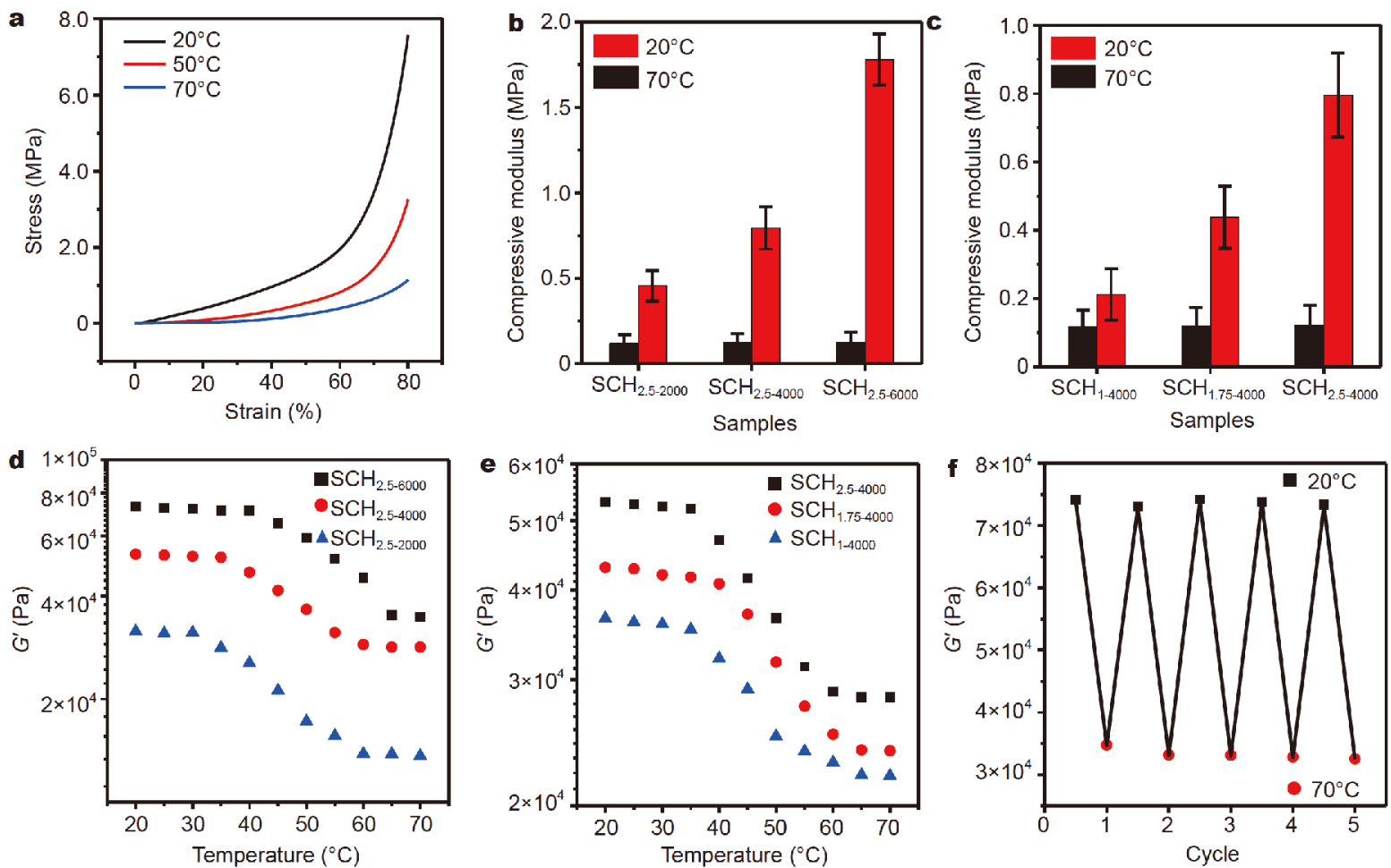

Figure 3 Thermomechanical properties of SCHs. (a) Compressive strain-stress curves of $\mathrm{SCH}_{2.5-6000}$ at different temperature. (b) Compressive modulus of SCHs with different molecular weights. (c) Compressive modulus of SCHs with different PCL contents. (d, e) The storage moduli ( $\left.G^{\prime}\right)$ of the SCHs with different PCL molecular weights and contents at a frequency $(\omega)$ sweep of $15.8 \mathrm{rad} \mathrm{s}^{-1}$ and a constant shear strain $(\gamma)$ of $0.5 \%$ by increasing temperature. (f) The storage moduli $\left(G^{\prime}\right)$ of the $\mathrm{SCH}_{2.5-6000}$ in a temperature circulating test.

during the swelling process, further indicating that the PCL side chains content can control the swelling degrees of the SCHs. Moreover, as shown in Fig. S5, the microstructures of the hydrogel with single hydrophilic framework and $\mathrm{SCH}_{2.5-4000}$ were observed by SEM, which showed that the porous nature of the samples. The pore size of the single hydrophilic network was $15 \mu \mathrm{m}$, and the $\mathrm{SCH}_{2.5-4000}$ had a smaller pore size of $7 \mu \mathrm{m}$. This demonstrates that the polymer network density of SCHs is obviously higher than the single hydrophilic framework, because of PCL side chains filling in the pore of hydrophilic hydrogel framework.

In our heterogeneous network structure of $\mathrm{SCH}$, semicrystalline PCL side chains aggregated to form rigid micro-domains, and hydrophilic backbones acted as elastic framework. We utilized the small angle X-ray scattering (SAXS) and wide angle X-ray scattering (WAXS) to further investigate the detail microstructures of the SCHs with different contents and molecular weights of PCL side chains. In our case, we utilized Teubner-Stray (T-S) model to analyze the SAXS data of the SCHs. The T-S model describes the correlation between scattering intensity $(I(q))$ and scattering vector $(q)$ and is expressed as Equation (S1) (Fig. 1d, Theoretical Section, Supplementary Information) [37]. The T-S model has been applied to analyze the scattering peaks of multiphase systems of polymers such as the microphase-separation systems formed by water, oil and surfactants, copolymer assembly systems, polymer blends and hydrogels with comb-type polymer network [38-42]. For example, the SAXS profile of $\mathrm{SCH}_{2.5-6000}$ exhibits a rather broad peak at $0.18 \mathrm{~nm}^{-1}$ in the intermediate $q$ range (Fig. 1d). Through nonlinear least-squares fitting of SAXS data in the correlation peak, the T-S model described a similar shape with a broad peak at $0.172 \mathrm{~nm}^{-1}$, indicating that our SAXS profiles of SCHs are fitted by the T-S model. This result confirmed that rigid semi-crystalline PCL domains uniformly dispersed in the elastic hydrophilic framework. As shown in Fig. 1e and Fig. S6, all the spectra of SCHs exhibit similar intensity profiles based on similar heterogeneous polymer network, indicating that all the SCHs exhibit similar microstructures. They show the SAXS profiles of the SCHs with different contents and molecular weights of PCL side chains, respectively. The scat- 
a
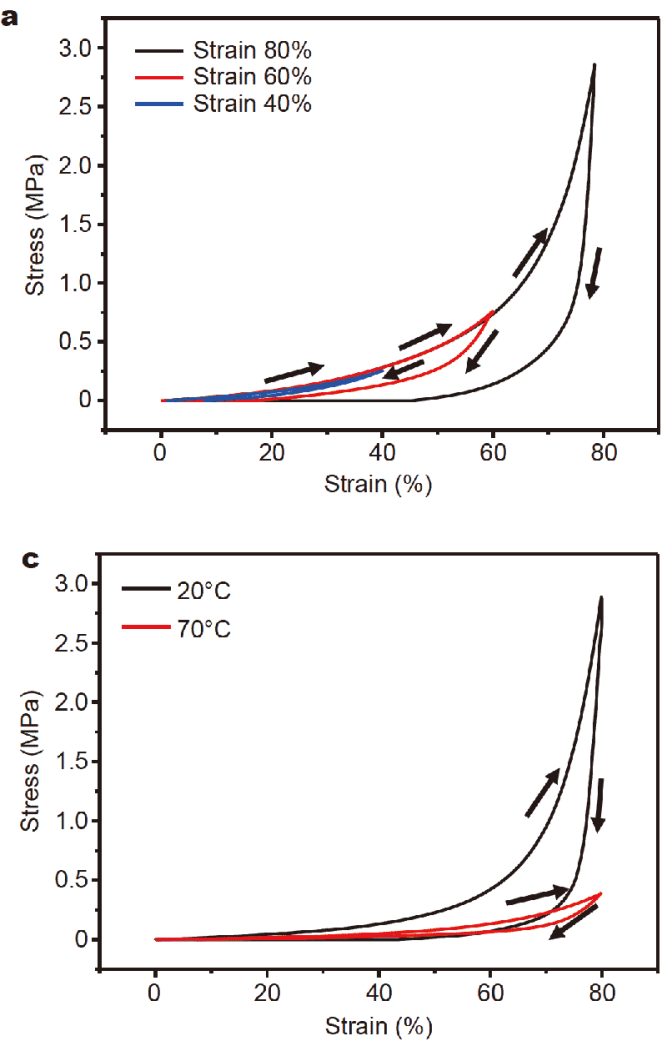

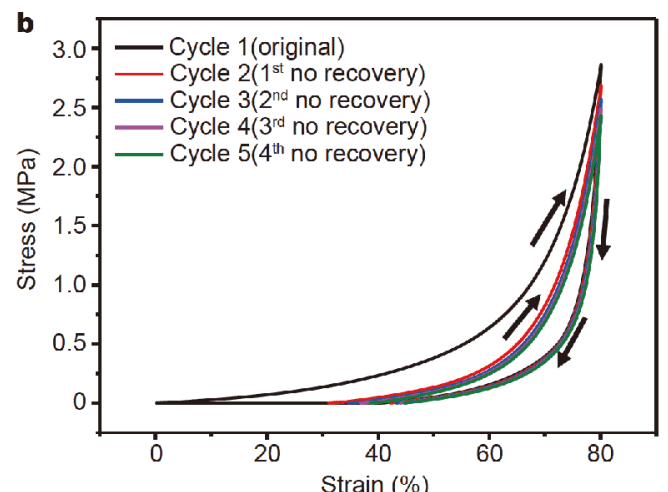

Recovery by heating

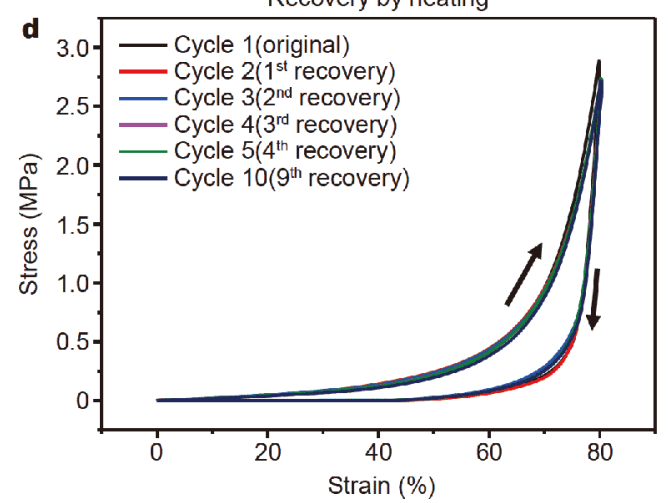

Figure 4 Self-recovery performance of SCHs. (a) The loading-unloading curves of the $\mathrm{SCH}_{2.5-4000}$ with different compressive strains at $20^{\circ} \mathrm{C}$. (b) The cyclic loading-unloading compressive tests of $\mathrm{SCH}_{2.5-4000}$ at $20^{\circ} \mathrm{C}$. (c) The loading-unloading compressive tests of $\mathrm{SCH}_{2.5-4000}$ at 20 and $70^{\circ} \mathrm{C}$. (d) $\mathrm{The}$ cyclic loading-unloading compressive tests of $\mathrm{SCH}_{2.5-4000}$ at $20^{\circ} \mathrm{C}$ with recovered by heat.

tering peak maximum $\left(q_{\max }\right)$ of hydrogels is associated with the scattering of interacting semi-crystalline PCL domains. According to Bragg's law, $d$ was calculated by $d=2 \pi / q_{\max }$, representing the average distance between PCL domains in hydrogels. With increasing the PCL contents from 1 to 2.5 , the peak maximum position slightly shifts to higher $q$ values, indicating that $d$ decreases from $33.7 \mathrm{~nm}$ to $28.7 \mathrm{~nm}$. It stems from the increased density of PCL domains in the hydrogel polymer network (which is proportional to $1 / d^{3}$ ), corresponding to the swelling behavior of the SCHs in which the swelling ratios decreases with increasing the PCL contents. The average distance between the PCL domains increased from 21.4 to $34.9 \mathrm{~nm}$ when increasing the PCL molecular weight from 2,000 to 6,000 (Fig. S6). In addition, as shown in Fig. S7, the WAXS profile of $\mathrm{SCH}_{2.5-6000}$ exhibits characteristic diffraction peaks at $2 \theta=21.2^{\circ}, 23.5^{\circ}$, corresponding to the (110) and (200) diffractions of PCL polymer, while other $\mathrm{SCH}$ s exhibit a broad diffraction peak. With increasing the molecular weight of PCL, the diffraction intensity of SCHs increases obviously, in- dicating that crystallinity of SCHs increases. Similarly, the diffraction intensity of SCHs increases slightly with increasing the PCL contents, demonstrating that the increased density of PCL domains enhances the crystallinity of SCHs.

\section{Mechanical properties of hydrogels}

Combining the rigid semi-crystalline PCL micro-domains with elastic hydrophilic framework, our SCHs exhibited high mechanical strength. We investigated the mechanical performance of SCHs through changing PCL molecular weights and contents. Fig. 2a shows the compressive stress-strain curves of $\mathrm{SCHs}$ with different molecular weights and hydrogel with P(DMA-co-MEA) hydrophilic network. All SCH samples exhibit high compressive strength (1.73-7.57 MPa). For example, the compressive strength of $\mathrm{SCH}_{2.5-6000}$ is $7.57 \mathrm{MPa}$, which demonstrates that the mechanical performance of hydrogel polymer network can be enhanced by the filling effect of the rigid semi-crystalline micro-domains. In contrast, the P(DMAco-MEA) hydrogel has a poor mechanical strength with 
0.24 MPa of compressive strength due to lack of the filling effect of the micro-domains (Fig. 2c). Thereby, the compressive modulus of $\mathrm{SCH}_{2.5-6000}(1.76 \mathrm{MPa})$ is approximately 35 times than the compressive modulus of the $\mathrm{P}$ (DMA-co-MEA) hydrogel $(0.05 \mathrm{MPa})$ due to the microphase-separation heterogeneous polymer network. With increasing the molecular weights of PCL side chains, the PCL domains possessed high crystallinity, which resulted in an increase of the filling effect of the micro-domains in the polymer network. Thereby, the compressive strength and compressive modulus of SCHs increased obviously. As shown in Fig. 2b, the compressive strengths of SCHs with different PCL contents $(1,1.75$, 2.5) are 1.50, 1.76 and $2.44 \mathrm{MPa}$, respectively. These SCHs have compressive modulus (0.21-0.80 MPa) with increasing the PCL contents from 1 to 2.5 (Fig. 2d). It is because that the density of the rigid semi-crystalline PCL domains increases caused by decreasing the average distance between the micro-domains with increasing the PCL contents. Thereby, the high density of the rigid micro-domains can efficiently enhance the mechanical performance of the SCHs.

\section{Thermomechanical properties of hydrogels}

The rigid semi-crystalline PCL domains within the elastic hydrophilic framework possess the melt-crystallization transition performance by thermal stimuli, which results in the excellent thermomechanical properties of SCHs. When the temperature was heated to $70^{\circ} \mathrm{C}$, the crystallization of PCL was melted and the rigid micro-domains became soft. Thereby, combining the elastic hydrophilic framework, the mechanical properties of our SCHs decreased with increasing temperature. In Fig. $3 \mathrm{a}$, the compressive strength of $\mathrm{SCH}_{2.5-6000}$ decreased obviously as temperature was increasing. For example, the compressive strength of $\mathrm{SCH}_{2.5-6000}$ is $7.57 \mathrm{MPa}$ at $80 \%$ and $20^{\circ} \mathrm{C}$, whereas the strength decreases to $3.26 \mathrm{MPa}$ at $55^{\circ} \mathrm{C}$, and lower to $1.14 \mathrm{MPa}$ at $70^{\circ} \mathrm{C}$ (Fig. S8). Furthermore, there are distinct differences between the compressive modulus of all SCHs at 20 and $70^{\circ} \mathrm{C}$, which demonstrates that the SCHs are stiff at the temperature lower than $T_{\mathrm{m}}$ and soft at the temperature above $T_{\mathrm{m}}$ (Fig. $3 \mathrm{~b}$ and c). The difference between compressive modulus at 20 and $70^{\circ} \mathrm{C}$ increased with increasing the molecular weights of PCL side chains. With increasing the molecular weight of PCL, the compressive modulus increased distinctly at $20^{\circ} \mathrm{C}$ due to the high crystallinity of micro-domains, while the compressive modulus increased slightly at $70^{\circ} \mathrm{C}$ because of the slight difference between the mechanical strength of the soft micro-domains. We further investigated the ther- momechanical properties of SCHs by utilizing the temperature sweep rheological measurements. Fig. $3 \mathrm{~d}$ and e reveal a significant decrease in $G^{\prime}$ with rising the temperature from 20 to $70^{\circ} \mathrm{C}$, indicating that the mechanical performance of SCHs decreased obviously as temperature increased due to the melt of the semi-crystalline PCL side chains in the rigid micro-domains. For example, the storage moduli $\left(G^{\prime}\right)$ of $\mathrm{SCH}_{2.5-6000}$ is $73 \mathrm{kPa}$ at $20^{\circ} \mathrm{C}$ and decreased to $35 \mathrm{kPa}$ at $70^{\circ} \mathrm{C}$. Meanwhile, the $G^{\prime}$ of the SCHs increased with increasing the molecular weights of PCL above $T_{\mathrm{m}}$ and below $T_{\mathrm{m}}$, which demonstrated that PCL micro-domains with high crystallinity can enhance the rheological properties. With increasing contents of PCL side chains, the storage moduli increased obviously due to the increase of the density of rigid micro-domains (Fig. S9). Furthermore, the storage modulus of $\mathrm{SCH}_{2.5-6000}$ remained stable at 20 and $70^{\circ} \mathrm{C}$ in the temperature circulating test, which demonstrates the stability of mechanical performance of SCHs during the melt-crystallization transition (Fig. 3f).

\section{Self-recoverable properties of hydrogels by thermal stimuli} In addition to the high mechanical properties and switchable mechanics, our SCHs displayed self-recovery property by thermal stimuli, which was studied by the loading-unloading compressive tests. For example, Fig. 4a shows the loading-unloading curves of the $\mathrm{SCH}_{2.5-4000}$ sample under different compressive strains at room temperature. With increasing the compressive strain, the hysteresis loop of $\mathrm{SCH}_{2.5-4000}$ increased dramatically, and the dissipated energy increased from $8 \mathrm{~kJ} \mathrm{~m}^{-3}$ (40\%) to $208 \mathrm{~kJ} \mathrm{~m}^{-3}$ (80\%) (Fig. S10a). $\mathrm{SCH}_{2.5-4000}$ exhibited such a distinct hysteresis loop when compressive strain was set to $80 \%$, indicating that heterogeneous polymer network with rigid micro-domains dispersed in hydrophilic framework could efficiently dissipate energy when they bore stress. In the successive loading-unloading compressive test without resting, the hysteresis loop of $\mathrm{SCH}_{2.5-4000}$ decreased sharply, revealing that the dissipated energy decreased from 208 (Cycle 1) to $112 \mathrm{~kJ} \mathrm{~m}^{-3}$ (Cycle 2) (Fig. $4 \mathrm{~b}$ ). It is because that rigid semi-crystalline PCL domains cannot recover to the original position owing to the lack of sufficient restoring stress from the elastic hydrophilic polymer framework under the large compressive strain stage. Moreover, the following loading-unloading cycles after first cycle exhibited almost constant hysteresis loop. It is shown that $\mathrm{SCH}_{2.5-4000}$ did not undergo more serious damage and exhibited good fatigue resistance. When the $\mathrm{SCH}_{2.5-4000}$ was heated to the temperature above $T_{\mathrm{m}}$ of the PCL, the rigid PCL domains became soft and elastic. 
a
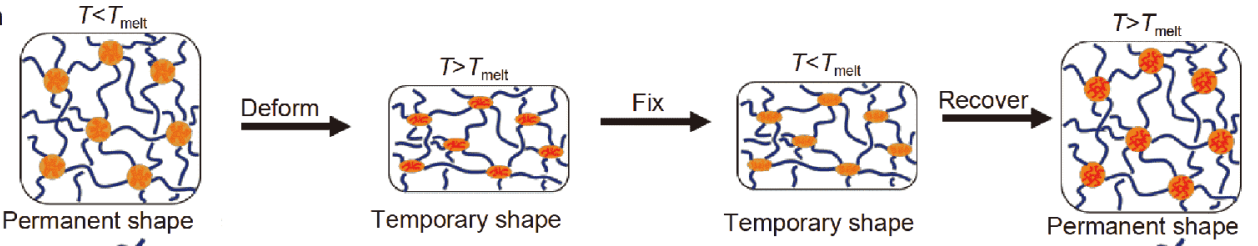

b

Temporary shape

Temporary shape
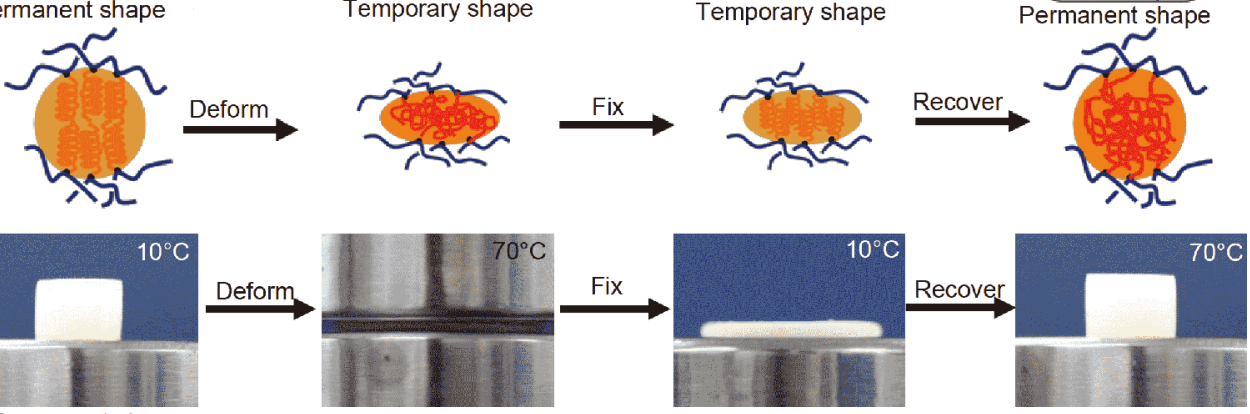

Permanent shape
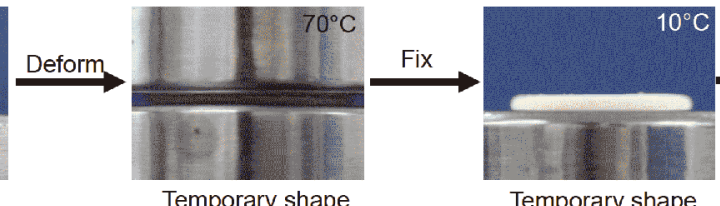

Temporary shape

Permanent shape
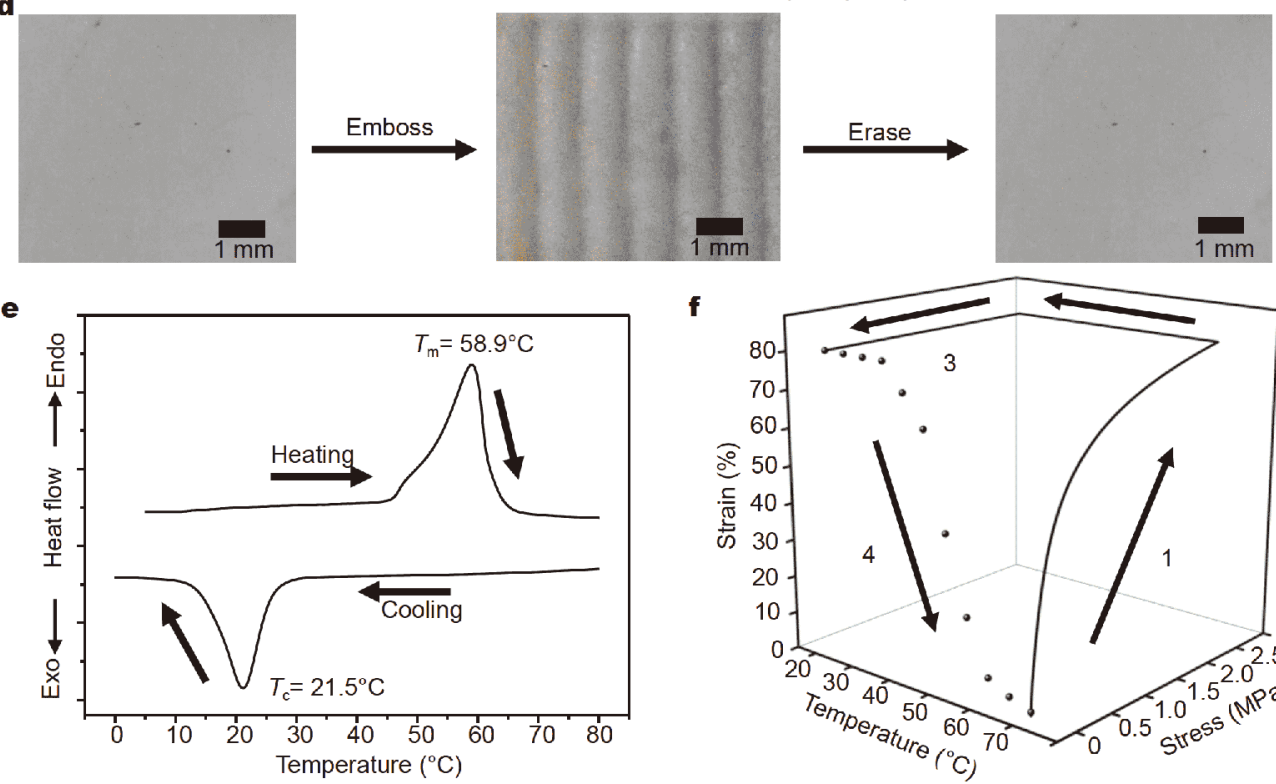

f

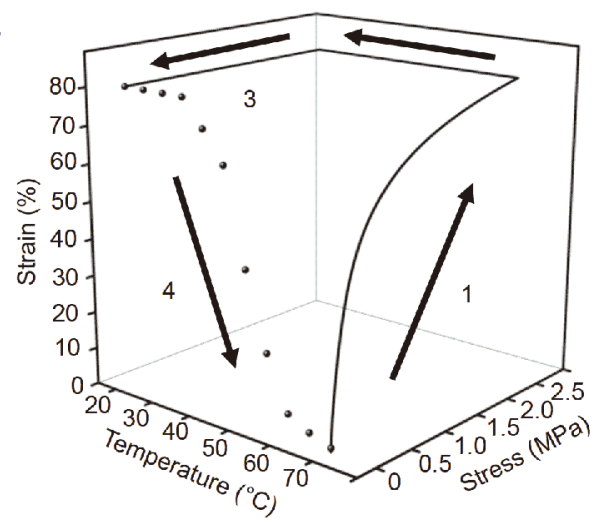

g
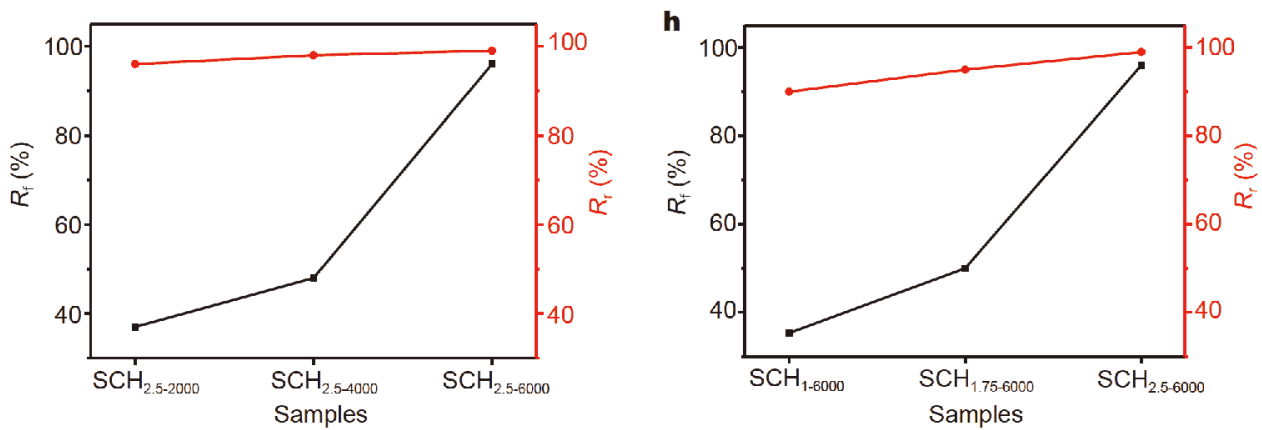

Figure 5 Shape memory behaviors of SCHs. (a) Schematic illustration of the shape memory behavior of SCHs in the process of deforming, fixing and recovering. (b) Schematic illustration of internal structure of the PCL domains in the shape memory process. (c) Images showing the shape memory effects of SCHs. (d) The surface microstructure embossing and erasing during the shape memory process. The scale bar is 1 mm. (e) DSC thermograms exhibiting the melting $\left(T_{\mathrm{m}}=58.9^{\circ} \mathrm{C}\right)$ and crystallization temperature $\left(T_{\mathrm{c}}=21.5^{\circ} \mathrm{C}\right)$ of SCHs samples. $(\mathrm{f})$ Shape memory behavior of compression and recovery. The black arrows indicate the four stages: (1) compressing at $70^{\circ} \mathrm{C}$; (2) cooling to $20^{\circ} \mathrm{C}$; $(3)$ uploaded; and (4) heating to $70^{\circ} \mathrm{C}$. (g) The fixed ratios and recovery ratios of SCHs with different molecular weights. (h) The fixed ratios and recovery ratios of SCHs with different PCL contents. 
Thereby, combining the elastic hydrophilic framework, the polymer network can provide enough resilience to achieve its self-recovery behavior. Compared to the residual strain $(45 \%)$ at $20^{\circ} \mathrm{C}$, the sample can fully recover its original shape at zero stress and $70^{\circ} \mathrm{C}$, and the hysteresis loop of $\mathrm{SCH}_{2.5-4000}$ at $70^{\circ} \mathrm{C}$ was much smaller than that at $20^{\circ} \mathrm{C}$ (Fig. 4c). It demonstrates that the soft and elastic hydrogel network can recover its shape at the temperature above the $T_{\mathrm{m}}$ of the PCL. In Fig. $4 \mathrm{~d}$, the $\mathrm{SCH}_{2.5-4000}$ exhibited stable mechanical strength and hysteresis loop during ten cyclic loading-unloading compressive tests at $20^{\circ} \mathrm{C}$, which recovered at $70^{\circ} \mathrm{C}$ between each cycle (Fig. S10b). The excellent self-recovery ability of SCHs has been shown through above loading-unloading curves by thermal stimuli.

\section{Shape memory properties}

Owing to the melt-crystallization in the PCL-MA domains and the stable network, we observed that the dry polymer network and $\mathrm{SCH}$ both exhibited shape memory effect. Fig. $5 \mathrm{a}$ and $\mathrm{b}$ illustrates the structure of the $\mathrm{SCHs}$ during the shape memory process. Above $T_{\mathrm{m}}$, the crystallization of the PCL domains was melted, which made the SCHs soften. The SCHs became easier to process and a temporary shape can be created by deformation. After lowering the temperature below $T_{\mathrm{m}}$, the PCL domains crystallized again and could fix the temporary shape of the SCHs. Later, the SCHs can recover their permanent shape by heating temperature above $T_{\mathrm{m}}$. Fig. $5 \mathrm{c}$ shows the shape memory behavior of the SCHs. The SCHs can compressively deform by up to $80 \%$ without mechanically fracturing above $T_{\mathrm{m}}$ and fix the temporary shape or recovery the permanent shape by thermal stimuli. Besides the shape memory at the macroscale, the deformation and recovery of surface features have attracted attention in the application of the microscale, such as micro-optics. Our SCHs also showed good ability on surface shape memory. Firstly, a structured stamp was brought in contact with the surface of SCHs when the SCHs were heated to the temperature above $T_{\mathrm{m}}$. Then, the patterns of ribbons were temporarily fixed on the material's surface by lowering the temperature below $T_{\mathrm{m}}$. And the patterns could be erased by heating the materials back above $T_{\mathrm{m}}$ to recovery their permanent shape (Fig. 5d).

In the permanent shape, the SCHs materials are at the lowest energy state (highest entropy) and the network of the SCHs is stable. The DSC curves of SCHs exhibited an endothermic peak at $58.9^{\circ} \mathrm{C}\left(T_{\mathrm{m}}\right)$ on heating and an exothermic peak at $21.5^{\circ} \mathrm{C}\left(T_{\mathrm{c}}\right)$ on cooling, resulting from the melt and crystallization of the PCL domains in the SCHs, respectively (Fig. 5e). When the SCHs were heated to the temperature above the $T_{\mathrm{m}}$, the crystallization of PCL domains was melted. Combining with the elastic framework, the SCHs were easy to change their permanent shape, which means raising the energy state of the materials. Then cooling the SCHs below the $T_{\mathrm{c}}$, the PCL domains were crystallized again, which served as the kinetic trap to maintain the materials in the high energy state. With heating the SCHs to the temperature above $T_{\mathrm{m}}$ again, the stored entropic energy is released and the mobility of polymer chains is restored, driving the elastic network back to the lowest energy state, and thus the materials accordingly recover the original shape. Fig. $5 f$ shows that the SCHs exhibit excellent shape memory effect. In the shape recovery process, the residual strain reduced with increasing the temperature. As shown in Fig. $5 \mathrm{~g}$ and $\mathrm{h}$, the shape fixity ratio $\left(R_{\mathrm{f}}\right)$ and shape recovery ratio $\left(R_{\mathrm{r}}\right)$ of $\mathrm{SCH}_{2.5-6000}$ were $96 \%$ and $98 \%$, respectively. All the $\mathrm{SCH}$ show the shape recovery ratio above $90 \%$, which increases with increasing the molecular weight or PCL contents. However, the shape fixity ratio of other samples except $\mathrm{SCH}_{2.5-6000}$ is lower than that of $\mathrm{SCH}_{2.5-6000}$ due to the lower crystallinity or fewer PCL domains. Furthermore, besides the SCHs, our dry polymer networks also exhibit good shape memory behavior. As shown in Fig. S12, the dry polymer networks can fix their temporary shape and recovery their permanent shape by thermal stimuli in the compressively deform process. We also can emboss and erase micropatterns in their surface by thermal stimuli. The excellent shape memory effect on the dry polymer network and SCHs can make our materials applied in complex environment. And the surface shape memory behaviors give our materials a wider potential application on switchable surface wettability, adhesion and actuator.

\section{CONCLUSIONS}

In summary, we reported stimuli-responsive $\mathrm{SCH}$ with high mechanical strength, thermomechanical performance, self-recovery property and shape memory behavior. In the heterogeneous polymer network, the PCL side chains aggregate to form the rigid micro-domains and hydrophilic backbones serve as the framework. By changing the molecular weights and contents of PCL side chains, we can tune the mechanical properties of our materials, such as compressive strength (1.50-7.57 MPa) and compressive modulus $(0.21-1.76 \mathrm{MPa})$. Due to the melt-crystallization transition of the PCL, the SCHs exhibit excellent thermomechanical performance and 
self-recovery properties by thermal stimuli. Meanwhile, our materials show excellent shape memory and surface micro-patterning behaviors. They can be used as smart materials in complex environments. Therefore, our comb-type hydrogels can serve as smart materials applied in many fields, such as tissue engineering, drug delivery and soft devices.

\section{Received 18 July 2018; accepted 31 August 2018; published online 21 September 2018}

1 Zhao ZG, Xu YC, Fang RC, et al. Bioinspired adaptive gel materials with synergistic heterostructures. Chin J Polym Sci, 2018, 36: 683696

2 Zhao Z, Fang R, Rong Q, et al. Bioinspired nanocomposite hydrogels with highly ordered structures. Adv Mater, 2017, 29: 1703045

3 Chen L, Yin Y, Liu Y, et al. Design and fabrication of functional hydrogels through interfacial engineering. Chin J Polym Sci, 2017, 35: 1181-1193

4 Zhang Y, Liao J, Wang T, et al. Polyampholyte hydrogels with $\mathrm{pH}$ modulated shape memory and spontaneous actuation. Adv Funct Mater, 2018, 28: 1707245

5 Kim YS, Liu M, Ishida Y, et al. Thermoresponsive actuation enabled by permittivity switching in an electrostatically anisotropic hydrogel. Nat Mater, 2015, 14: 1002-1007

6 Lu W, Le X, Zhang J, et al. Supramolecular shape memory hydrogels: a new bridge between stimuli-responsive polymers and supramolecular chemistry. Chem Soc Rev, 2017, 46: 1284-1294

7 Le X, Lu W, Zheng J, et al. Stretchable supramolecular hydrogels with triple shape memory effect. Chem Sci, 2016, 7: 6715-6720

8 Li Z, Lu W, Ngai T, et al. Mussel-inspired multifunctional supramolecular hydrogels with self-healing, shape memory and adhesive properties. Polym Chem, 2016, 7: 5343-5346

9 Wang W, Fan X, Li F, et al. Magnetochromic photonic hydrogel for an alternating magnetic field-responsive color display. Adv Opt Mater, 2018, 6: 1701093

10 Shim TS, Kim SH, Sim JY, et al. Dynamic modulation of photonic bandgaps in crystalline colloidal arrays under electric field. Adv Mater, 2010, 22: 4494-4498

11 Klouda L, Mikos AG. Thermoresponsive hydrogels in biomedical applications. Eur J Pharm BioPharm, 2008, 68: 34-45

12 Gao F, Zhang Y, Li Y, et al. Sea cucumber-inspired autolytic hydrogels exhibiting tunable high mechanical performances, repairability, and reusability. ACS Appl Mater Interfaces, 2016, 8: 89568966

13 Hu Y, Kahn JS, Guo W, et al. Reversible modulation of DNA-based hydrogel shapes by internal stress interactions. J Am Chem Soc, 2016, 138: 16112-16119

14 Dong LC, Yan Q, Hoffman AS. Controlled release of amylase from a thermal and $\mathrm{pH}$-sensitive, macroporous hydrogel. J Control Release, 1992, 19: 171-177

15 Zhang XZ, Yang YY, Chung TS, et al. Preparation and characterization of fast response macroporous $\operatorname{poly}(\mathrm{N}$-isopropylacrylamide) hydrogels. Langmuir, 2001, 17: 6094-6099

16 Rong Q, Lei W, Chen L, et al. Anti-freezing, conductive selfhealing organohydrogels with stable strain-sensitivity at subzero temperatures. Angew Chem Int Ed, 2017, 56: 14159-14163
17 Luo F, Sun TL, Nakajima T, et al. Oppositely charged polyelectrolytes form tough, self-healing, and rebuildable hydrogels. Adv Mater, 2015, 27: 2722-2727

18 Zhang HJ, Sun TL, Zhang AK, et al. Tough physical double-network hydrogels based on amphiphilic triblock copolymers. Adv Mater, 2016, 28: 4884-4890

19 Zhao Z, Zhang K, Liu Y, et al. Highly stretchable, shape memory organohydrogels using phase-transition microinclusions. Adv Mater, 2017, 29: 1701695

20 Dai X, Zhang Y, Gao L, et al. A mechanically strong, highly stable, thermoplastic, and self-healable supramolecular polymer hydrogel. Adv Mater, 2015, 27: 3566-3571

21 Zhang Y, Li Y, Liu W. Dipole-dipole and H-bonding interactions significantly enhance the multifaceted mechanical properties of thermoresponsive shape memory hydrogels. Adv Funct Mater, 2015, 25: 471-480

22 Liu M, Jiang L. Dialectics of nature in materials science: binary cooperative complementary materials. Sci China Mater, 2016, 59: 239-246

23 Zhao Z, Liu Y, Zhang K, et al. Biphasic synergistic gel materials with switchable mechanics and self-healing capacity. Angew Chem, 2017, 129: 13649-13654

24 Gao H, Zhao Z, Cai Y, et al. Adaptive and freeze-tolerant heteronetwork organohydrogels with enhanced mechanical stability over a wide temperature range. Nat Commun, 2017, 8: 15911

25 Abdurrahmanoglu S, Can V, Okay O. Design of high-toughness polyacrylamide hydrogels by hydrophobic modification. Polymer, 2009, 50: 5449-5455

26 Tuncaboylu DC, Argun A1, Sahin M, et al. Structure optimization of self-healing hydrogels formed via hydrophobic interactions. Polymer, 2012, 53: 5513-5522

27 Bilici C, Ide S, Okay O. Yielding behavior of tough semicrystalline hydrogels. Macromolecules, 2017, 50: 3647-3654

28 Zhang Z, Ni J, Chen L, et al. Biodegradable and thermoreversible PCLA-PEG-PCLA hydrogel as a barrier for prevention of postoperative adhesion. Biomaterials, 2011, 32: 4725-4736

29 Yu L, Zhang H, Ding J. A subtle end-group effect on macroscopic physical gelation of triblock copolymer aqueous solutions. Angew Chem Int Ed, 2006, 45: 2232-2235

30 Chen L, Ci T, Yu L, et al. Effects of molecular weight and its distribution of PEG block on micellization and thermogellability of PLGA-PEG-PLGA copolymer aqueous solutions. Macromolecules, 2015, 48: 3662-3671

31 Chen L, Li X, Cao L, et al. An injectable hydrogel with or without drugs for prevention of epidural scar adhesion after laminectomy in rats. Chin J Polym Sci, 2016, 34: 147-163

32 Cui SQ, Yu L, Ding JD. Injectable thermogels based on block copolymers of appropriate amphiphilicity. Acta Polym Sin, 2018: 997-1015

33 Bellin I, Kelch S, Langer R, et al. Polymeric triple-shape materials. Proc Natl Acad Sci USA, 2006, 103: 18043-18047

34 Lendlein A, Langer R. Biodegradable, elastic shape-memory polymers for potential biomedical applications. Science, 2002, 296: 1673-1676

35 Huang M, Zhao K, Wang L, et al. Dual stimuli-responsive polymer prodrugs quantitatively loaded by nanoparticles for enhanced cellular internalization and triggered drug release. ACS Appl Mater Interfaces, 2016, 8: 11226-11236

36 Yang CS, Wu HC, Sun JS, et al. Thermo-induced shape-memory PEG-PCL copolymer as a dual-drug-eluting biodegradable stent. 
ACS Appl Mater Interfaces, 2013, 5: 10985-10994

37 Teubner M, Strey R. Origin of the scattering peak in microemulsions. J Chem Phys, 1987, 87: 3195-3200

38 Peng S, Guo Q, Hughes TC, et al. In situ synchrotron SAXS study of polymerizable microemulsions. Macromolecules, 2011, 44: 3007-3015

39 Wu G, Ying Q, Chu B. Lamellar structure of block copolymer poly (oxyethylene-oxypropylene-oxyethylene) in xylene/water mixtures. Macromolecules, 1994, 27: 5758-5765

40 Washburn NR, Lodge TP, Bates FS. Ternary polymer blends as model surfactant systems. J Phys Chem B, 2000, 104: 6987-6997

41 Cao H, Chang X, Mao H, et al. Stereocomplexed physical hydrogels with high strength and tunable crystallizability. Soft Matter, 2017, 13: 8502-8510

42 Guo H, Mussault C, Brûlet A, et al. Thermoresponsive toughening in LCST-type hydrogels with opposite topology: from structure to fracture properties. Macromolecules, 2016, 49: 4295-4306
Acknowledgements This work was financially supported by the National Natural Science Foundation of China (21574004), the National Natural Science Funds for Distinguished Young Scholar (21725401), the Fundamental Research Funds for the Central Universities, the National 'Young Thousand Talents Program', and the China Postdoctoral Science Foundation (2017M620012).

Author contributions Zhang $\mathrm{K}$, Zhao $\mathrm{Z}$ and Liu $\mathrm{M}$ designed the project. Zhang $\mathrm{K}$ and Huang J preformed the experiments. Zhang $\mathrm{K}$, Zhao Z, Zhao T and Fang R analyzed the results. Zhang K, Fang R and Liu $M$ wrote the paper. All authors contributed to general discussion of the article

Conflict of interest The authors declare that they have no conflict of interest.

Supplementary information Experimental details and supporting data are available in the online version of the paper.

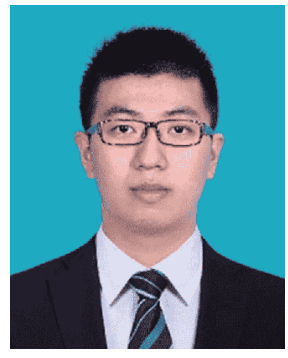

Kangjun Zhang received his BSc degree from Beihang University in 2016. He is now a master candidate under the supervision of Prof. Mingjie Liu at Beihang University. His research focuses on the fabrication of smart hydrogels based on cooperative complementary interaction.
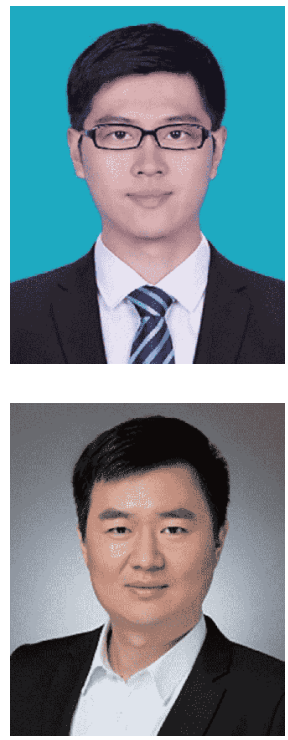

Ruochen Fang is a post-doctor in Beihang University. In 2011, He received his BSc degree in polymer materials and engineering in the Department of Chemistry from Jilin University. Then he joined Prof. Xi Zhang's group and received his $\mathrm{PhD}$ degree from Tsinghua University in 2016. His current research interest focuses on binary cooperative complementary nanomaterials and bioinspired material science.

\section{热响应性自恢复、形状记忆半结晶水凝胶}

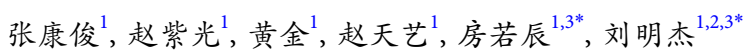

摘要 热响应性水凝胶在组织工程、药物运输和柔性器件等领域有着广泛应用. 但传统的热响应性水凝胶在大形变下回复性能差, 而且 一般需要复杂的化学合成. 亲疏水二元协同作用可以使水凝胶网络具备优异的响应性和力学性能. 基于此, 我们制备了具有异质梳状网络 的半结晶水凝胶. 这种半结晶水凝胶以亲水的DMA和MEA作为网络框架, 疏水的PCL聚集形成结晶微区作为热响应单元, 表现出优异的 力学性质、自恢复特性、热响应性机械性能和形状记忆的能力. 